\title{
Análisis de la huella plantar en escolares de 8 a 10 años
}

\author{
Analysis of the footprint in school age of 8-10 years old
}

\author{
María Victoria Giraldo Mateos ${ }^{1}$, Patricia Palomo López ${ }^{2 .}$ \\ ${ }^{1}$ Grado en Podología por la Universidad de Extremadura. \\ ${ }^{2}$ Doctora en Podología por la Universidad Rey Juan Carlos. Profesor Colaborador. Universidad de Extremadura. \\ patibiom@unex.es
}

\author{
Correspondencia: \\ María Victoria Giraldo Mateos. Grado en Podología por la Universidad de Extremadura. \\ C/Vistahermosa. No70, $1^{\circ}$ izqda. \\ E-06200 Almendralejo (Badajoz). \\ Correo electrónico: mavy_92@hotmail.com
}

Fecha de recepción: 9 julio de 2015

Fecha de aceptación: 14 de octubre de 2015

Los autores declaran no tener ningún tipo de interés económico o comercial.

\section{RESUMEN}

Los problemas biomecánicos en los niños, es un tema de mucho auge en la actualidad, existiendo controversia en distintos ámbitos como son, la presencia de huella aplanada en la mayor parte de los niños o la hiperlaxitud articular ligada a dolor musculoesquelético.

El objetivo del estudio fue determinar qué tipo de huella plantar es más frecuente en niños de 8 a 10 años escolarizados en el área de Plasencia. Para ello se tomó como muestra 50 niños, de los cuales 28 eran varones y 22 hembras. A todos los sujetos del estudio se les realizó una evaluación de la huella plantar en estática, así como una exploración de distintos parámetros a través de la inspección en bipedestación (fórmula digital, retropié).

Así, los resultados muestran que la huella excavada está presente en un 72\% de los casos de la población. El 16\% era perteneciente a una huella excavada en la que encontramos relación con un mayor porcentaje de peso.

Para la fórmula digital encontramos que la más frecuente es el pie egipcio en un $40 \%$ de los casos y que el predominio en cuanto al retropié, es un retropié normal.

Con respecto a la hiperlaxitud articular comprobamos que es más frecuente en niñas y que ninguna de ellas presenta una asociación al dolor musculoesquelético.

Como línea futura podría establecerse un estudio más exhaustivo con nuevas técnicas y valorando la estática y la dinámica del niño, para así tener un estudio más exacto de las diferentes variables estudiadas en la muestra de población.

Palabras claves: edad escolar; huella plantar; pie; plataforma de presiones.

\footnotetext{
ABSTRACT

Biomechanical problems in children, is an important subject currently, existing controversy in different areas, for example, the majority of children have a flattened footprint, or the hypermobility joint is linked to a musculoskeletal pain.

The objective of the study was to determine what kind of footprint is most frequent in school-age children (8-10 years) in the area of Plasencia. This was taken as a sign 50 children, of whom 28 were males and 22 females.

All the subjects in the study underwent an assessment of footprint planted in static as well as an exploration of different parameters through inspection in a standing position (formula digital, rearfoot).

The results show that excavated footprint is present in a $72 \%$ cases of the population, $16 \%$ was belonging to an excavated footprint in which we find a higher percentage of weight related.

For the digital formula we find that the most common is the Egyptian foot by $40 \%$ of the cases and that the prevalence in the rearfoot, is a normal hindfoot.

In relation with the hypermobility joint, we check that it is more common in girls and that none of them presents an association to musculoskeletal pain.
} 
As a future line we could establish a more comprehensive study with new techniques and valuingchild's statics and dynamics, to have a more accurate study of the different variables in the sample population studied.

Key words: foot; school-age; footprint; pressure platform.

Referencia normalizada: Giraldo Mateos MV, Palomo López P. Análisis de la huella plantar en escolares de 8 a 10 años. Rev. Int. Cienc. Podol. 2016; 10(2): 70-84.

Sumario: 1. Introducción. 2. Objetivos. 3. Material y métodos. 4. Resultados. 5. Discusión. 6. Conclusiones. Bibliografía. 


\section{INTRODUCCION}

El pie es un elemento fundamental para la comprensión de los mecanismos del paso formando parte inseparable en la función de apoyo, proporción y sostén del sistema humano. Por su localización está destinado a recibir toda la carga del cuerpo. Sirve de soporte al peso corporal durante el apoyo estático o en la fase de apoyo de la marcha. No se trata únicamente de una plataforma de apoyo estable y sólido, sino que también se adapta a la forma y relieve de la superficie ${ }^{1-4}$.

También se define como una obra arquitectónica compleja que coordina 107 ligamentos, 19 músculos intrínsecos, con 26 huesos y es esencial para la posición bípeda humana ${ }^{5,6}$.

El empleo de nuevas tecnologías tiene una gran importancia en el estudio del movimiento, tanto en sujetos normales como en personas que sufran diversas patologías o traumatismos y sus capacidades motrices se vean modificadas. También puede aplicarse a la neurología, rehabilitación, fabricación del calzado o campo deportivo ${ }^{1,7}$.

El ser humano necesita conseguir una postura estable en bipedestación antes de iniciar la marcha ${ }^{1}$.

La marcha se hace estable en el niño a partir de los 3 o 4 años, por lo que a partir de esa edad podemos fijarnos con mayor atención en cualquier alteración de la marcha, desviaciones, desgaste del calzado, etc., que nos hagan sospechar que algo no funciona correctamente. Entre los 7 y 9 años, el niño consigue un patrón de marcha semejante al adulto ${ }^{1,8,9}$.

La hiperlaxitud articular, de mayor prevalencia en niños que en adultos, ha sido relacionada con la aparición de dolor musculoesquelético, aunque esta asociación se encuentra en discusión en los niños ${ }^{10-12}$.

El peso corporal se ha estudiado como factor de influencia en el desarrollo de los miembros inferiores en los niños, pues el sobrepeso incrementa los problemas del pie influyendo en las fuerzas verticales principalmente ${ }^{2,13}$.

La baropodometría abarca el estudio de las presiones generadas en el pie, facilitando la comprensión de los mecanismos mecánicos que influyen en la formación del pie del niño y su desarrollo ${ }^{9,14}$.
El uso de este método de evaluación de la huella plantar tiene ventajas como: bajo coste, facilidad de uso, no es invasivo, es repetible y cuantificable ${ }^{14,15}$.

\section{OBJETIVOS}

\subsection{Objetivo General}

Comprobar qué huella plantar es más frecuente en niños de 8 a 10 años escolarizados en el área de Plasencia.

\subsection{Objetivos Específicos}

- Comprobar si existe influencia del peso en la huella plantar de cada niño.

- Comprobar si utilizan un buen calzado para las distintas actividades a realizar.

- Ver cómo presentan el retropié.

- Comprobar qué tipo de fórmula digital es más frecuente.

- Comprobar si existe relación entre la huella plantar y Línea de Helbing.

- Averiguar la frecuencia de hiperlaxitud en edad comprendida de 8 a 10 años.

- Si existe diferencia en cuanto al sexo.

- Comprobar si los sujetos con hiperlaxitud articular presentan dolor musculoesquelético.

\section{MATERIAL Y MÉTODOS}

\section{Descripción de la muestra}

La muestra de este trabajo ha estado constituida por 50 niños, de los cuales 28 eran varones y 22 hembras, con edades comprendidas entre 8 y10 años.

Los individuos que han participado en el estudio proceden del colegio Alfonso VIII de $4^{\circ}$ de primaria en la localidad de Plasencia.

Los criterios de inclusión de la muestra han sido:

- Previa autorización de los padres o tutores legales.

- Tener una edad comprendida entre los 8 y 10 años.

- Que estén escolarizados.

\section{Procedimiento}

En primer lugar se realizó un informe y autorización para cada alumno, con el fin de in- 
formar a los padres y que dieran su consentimiento.

En el estudio se le realiza a cada niño una serie de mediciones que serán útiles para llevar a cabo los diferentes objetivos (edad, peso, número de pie).

Sobre el calzado, se recogen datos sobre su número o medida, el tipo y el desgaste sufrido. También se registra si existe laxitud a través de los criterios de Beighton.

Una vez recogido los diferentes datos, se les inspecciona en bipedestación para valorar el retropié trazando la Línea de Helbing en cada sujeto y valorar la fórmula digital.

Por último, le observamos la huella plantar en estática.

Las instrucciones a seguir para la plataforma fueron:

1. Posición anatómica en Cadena Cinética Cerrada.

2. En ángulo y base de sustentación o stand, tras caminar sobre la plataforma 30 segundos.

3. Permanecer estáticos mientras se van procesando los distintos fotogramas de las huellas.

\section{Equipamiento}

- Plataforma de presiones Podoprint de la marca Namrol.

- Báscula.

- Ordenador portátil.

- Cámara compact-réflex digital Sony alfa 200/tarjeta de memoria compact flash de 16 GB.

- Cinta métrica.

- Goniómetro de Moltgen.

- Sistema operativo Microsoft Word 2013.

- Para datos estadísticos se utiliza el software SPSS V15.

- Para imágenes utilizamos el programa PhotoScape 3.6.5.

\section{RESULTADOS}

En la población estudiada observamos que la mayor parte de los niños pesan alrededor de los 30-40 kilos. Excepto 3 casos en los que el peso era superior a 60 kilogramos (Figura 1).

Con referencia a la huella plantar (Tabla 1), obtenemos que la mayor parte de la población estudiada presentan una huella excavada, y que

\section{Histograma}

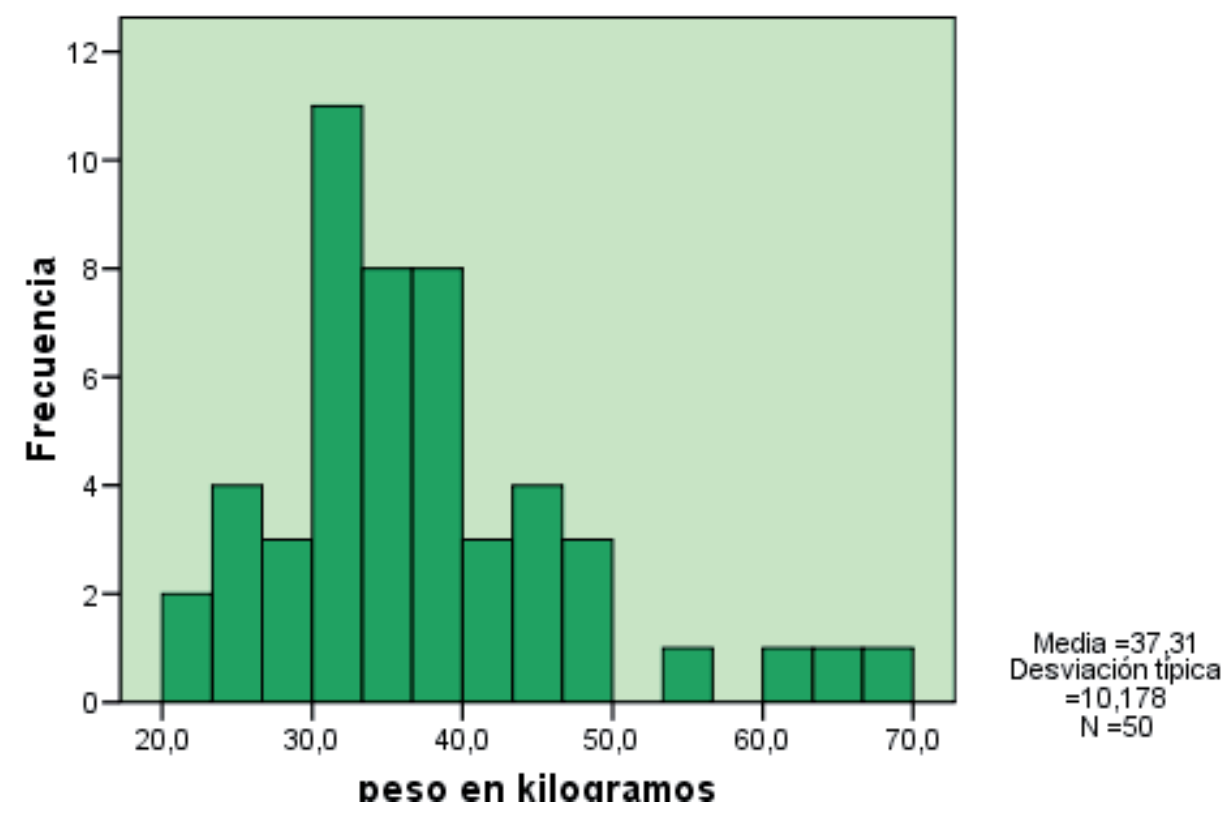

Figura 1. Histograma que refleja el peso de los niños recogidos en el estudio. 
solo el $12 \%$ de nuestra población tienen una huella normal (Figura 2).

Al estudiar el tipo de calzado y su sujeción (Tabla 2) pudimos observar que en niños el calzado más predominante era la zapatilla deportiva y en niñas las botas. Respecto a la contención-sexo, llegamos a la conclusión que la mayor parte de la población no tienen un calzado apropiado respecto a contención (Figura 3).

Tabla 1. Recuento sobre el tipo de huella plantar existente en los niños estudiados.

\begin{tabular}{lll} 
& Frecuencia & Porcentaje \\
\hline Aplanada & 8 & $16 \%$ \\
\hline Excavada & 36 & $72 \%$ \\
\hline Normal & 6 & $12 \%$ \\
Total & 50 & $100 \%$ \\
\hline
\end{tabular}

Tabla 2. Comprobación de la variante sexo con la sujeción del calzado.

\begin{tabular}{|c|c|c|c|c|c|}
\hline & Valor & gl & $\begin{array}{l}\text { Sig. asintótica } \\
\text { (bilateral) }\end{array}$ & $\begin{array}{l}\text { Sig. exacta } \\
\text { (bilateral) }\end{array}$ & $\begin{array}{l}\text { Sig. exacta } \\
\text { (unilateral) }\end{array}$ \\
\hline Chi-cuadrado de Pearson &, $034^{b}$ & 1 &, 854 & & \\
\hline $\begin{array}{l}\text { Corrección gor } \\
\text { continuidad }\end{array}$ &, 000 & 1 & 1,000 & & \\
\hline Razón de verosimilitudes &, 034 & 1 & 854 & & \\
\hline $\begin{array}{l}\text { Estadistico exacto de } \\
\text { Fisher }\end{array}$ & & & & 1,000 &, 540 \\
\hline N de casos válidos & 50 & & & & \\
\hline
\end{tabular}

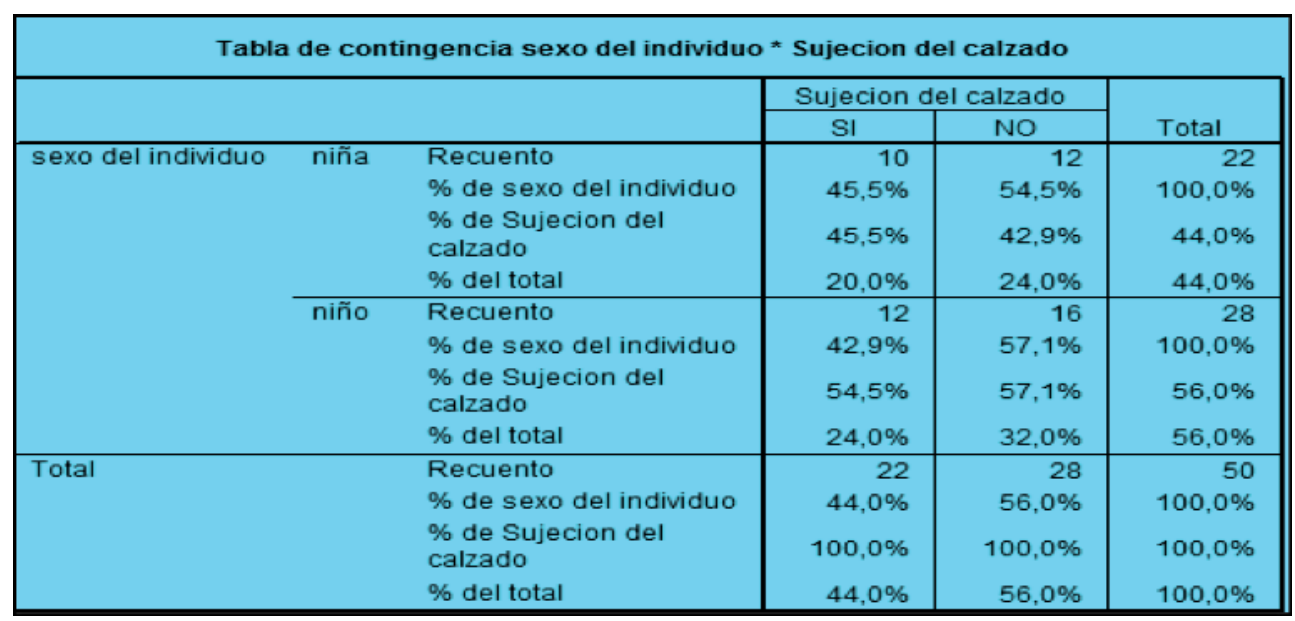




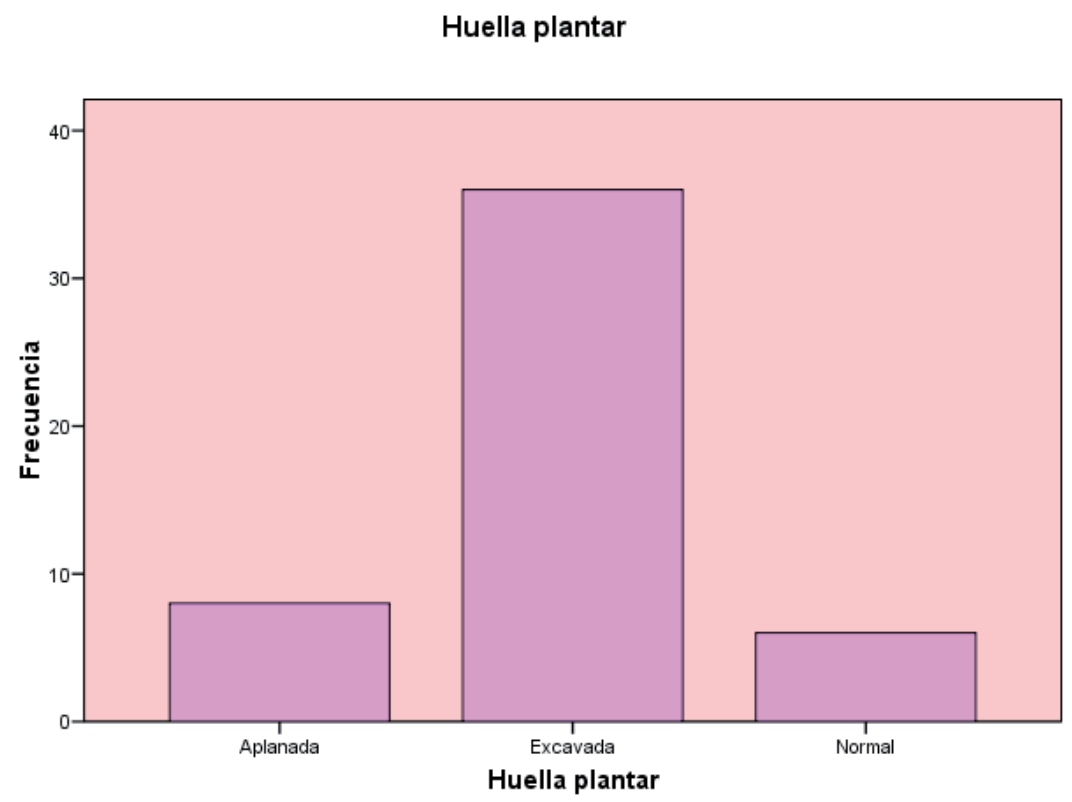

Figura 2. Frecuencia de las distintas huellas plantares.

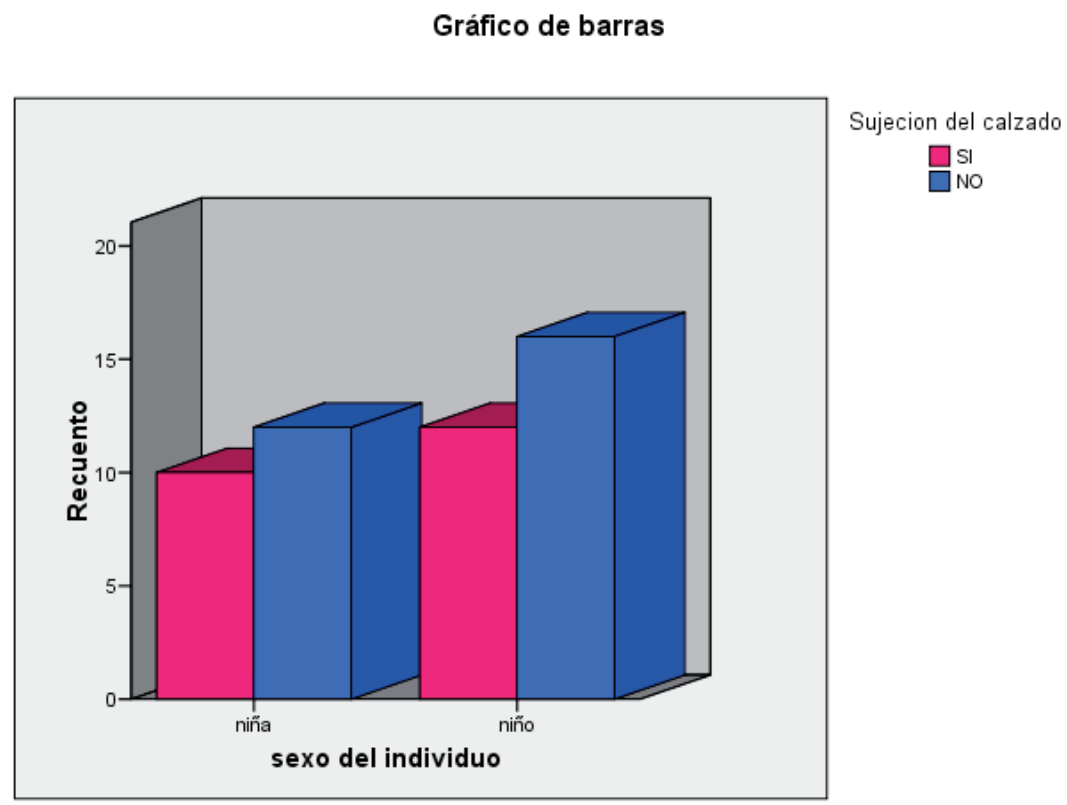

Figura 3. Representación gráfica de la distribución de sexo comparado con la sujeción del calzado. 
La fórmula digital con mayor predominancia (Figura 4) en el estudio, es el pie egipcio con un $40 \%$ de los casos. Seguido de un pie cuadrado con el $34 \% \mathrm{y}$, finalmente, el pie griego con el $26 \%$ de los niños.

Atendiendo a la Línea de Helbing concluimos que 28 de los 50 niños estudiados presentan un retropié normal, seguido de 20 usuarios con un retropié valgo y sólo 2 casos con un retropié varo (Tabla 3 ).

Comparando la variable Línea de Helbing \& huella plantar (Tabla 4) observamos que gran parte del estudio poseía un retropié normal con una huella excavada (Figura 5).

Al estudiar la laxitud ligamentosa en la población (Tabla 5), comprobamos que sólo el $16 \%$ de la población tenía dicha característica.

Para comprobar lo que apuntaban otros autores en cuanto a la prevalencia de la laxitud con referencia al sexo, realizamos un estudio comparativo entre los distintos sexos del estudio (Tabla 6). Los datos obtenidos fueron para niñas un $22,7 \%$ y para niños un $10,7 \%$, así pudimos afirmar que es más prevalente en el género femenino (Figura 6).

\section{DISCUSIÓN}

Al estudiar la fórmula digital encontramos que el pie egipcio lo presenta el $40 \%$ de los escolares, el pie cuadrado un $34 \%$ y el pie griego un $26 \%$.

Sin embargo, con la variable "Sexo" la predominancia del pie egipcio varía obteniéndose en niñas la máxima frecuencia de pie cuadrado con un $45,4 \%$ frente al pie egipcio en niños en un $42,8 \%$.

\section{Formula digital}

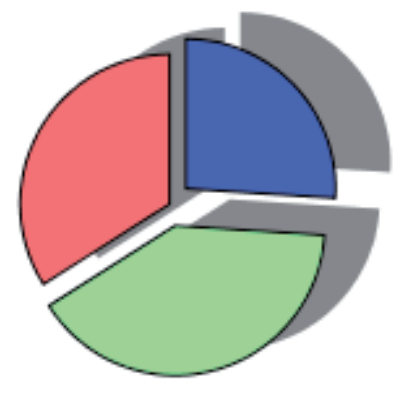

Figura 4. Fórmulas digitales del estudio.

Tabla 3. 28 de los 50 niños estudiados podemos verificar que presentan un retropié normal, seguido de 20 usuarios con un retropié valgo y sólo 2 casos con un retropié varo.

\begin{tabular}{cccc}
\multirow{4}{*}{ Válidos } & & Frecuencia & Porcentaje \\
& Normal & 28 & 56,0 \\
& Varo & 2 & 4,0 \\
& Valgo & 20 & 40,0 \\
& Total & 50 & 100,0 \\
\hline
\end{tabular}


Tabla 4. Comparación de las variables de huella plantar y Línea de Helbing.

\begin{tabular}{|c|c|c|c|c|c|c|}
\hline & & & \multicolumn{3}{|c|}{ Huella plantar } & \multirow[b]{2}{*}{ Total } \\
\hline & & & Aplanada & Excavada & Normal & \\
\hline \multirow{12}{*}{$\begin{array}{l}\text { Linea de } \\
\text { Helbing }\end{array}$} & Normal & Recuento & 6 & 21 & 1 & 28 \\
\hline & & $\%$ de Linea de Helbing & $21,4 \%$ & $75,0 \%$ & $3,6 \%$ & $100,0 \%$ \\
\hline & & $\%$ de Huella plantar & $75,0 \%$ & $58,3 \%$ & $16,7 \%$ & $56,0 \%$ \\
\hline & & $\%$ del total & $12,0 \%$ & $42,0 \%$ & $2,0 \%$ & $56,0 \%$ \\
\hline & Varo & Recuento & 0 & 2 & 0 & 2 \\
\hline & & $\%$ de Linea de Helbing & $.0 \%$ & $100,0 \%$ &, $0 \%$ & $100,0 \%$ \\
\hline & & $\%$ de Huella plantar & $.0 \%$ & $5,6 \%$ &, $0 \%$ & $4,0 \%$ \\
\hline & & $\%$ del total & $.0 \%$ & $4,0 \%$ &, $0 \%$ & $4,0 \%$ \\
\hline & Valgo & Recuento & 2 & 13 & 5 & 20 \\
\hline & & $\%$ de Linea de Helbing & $10,0 \%$ & $65,0 \%$ & $25,0 \%$ & $100,0 \%$ \\
\hline & & $\%$ de Huella plantar & $25,0 \%$ & $36,1 \%$ & $83,3 \%$ & $40,0 \%$ \\
\hline & & $\%$ del total & $4,0 \%$ & $26,0 \%$ & $10,0 \%$ & $40,0 \%$ \\
\hline \multirow[t]{4}{*}{ Total } & & Recuento & 8 & 36 & 6 & 50 \\
\hline & & $\%$ de Linea de Helbing & $16,0 \%$ & $72,0 \%$ & $12,0 \%$ & $100,0 \%$ \\
\hline & & $\%$ de Huella plantar & $100,0 \%$ & $100,0 \%$ & $100,0 \%$ & $100,0 \%$ \\
\hline & & $\%$ del total & $16,0 \%$ & $72,0 \%$ & $12,0 \%$ & $100,0 \%$ \\
\hline
\end{tabular}

Pruebas de chi-cuadrado

\begin{tabular}{|c|c|c|c|}
\hline & Valor & gl & $\begin{array}{l}\text { Sig. asintótica } \\
\text { (bilateral) }\end{array}$ \\
\hline Chi-cuadrado de Pearson & $6,389^{a}$ & 4 & .172 \\
\hline Razón de verosimilitudes & 6,911 & 4 & 141, \\
\hline $\begin{array}{l}\text { Asociación lineal por } \\
\text { lineal }\end{array}$ & 4,444 & 1 & ,035 \\
\hline N de casos válidos & 50 & & \\
\hline
\end{tabular}

a. 7 casillas $(77,8 \%)$ tienen una frecuencia esperada inferior a 5 . La frecuencia mínima esperada es ,24.

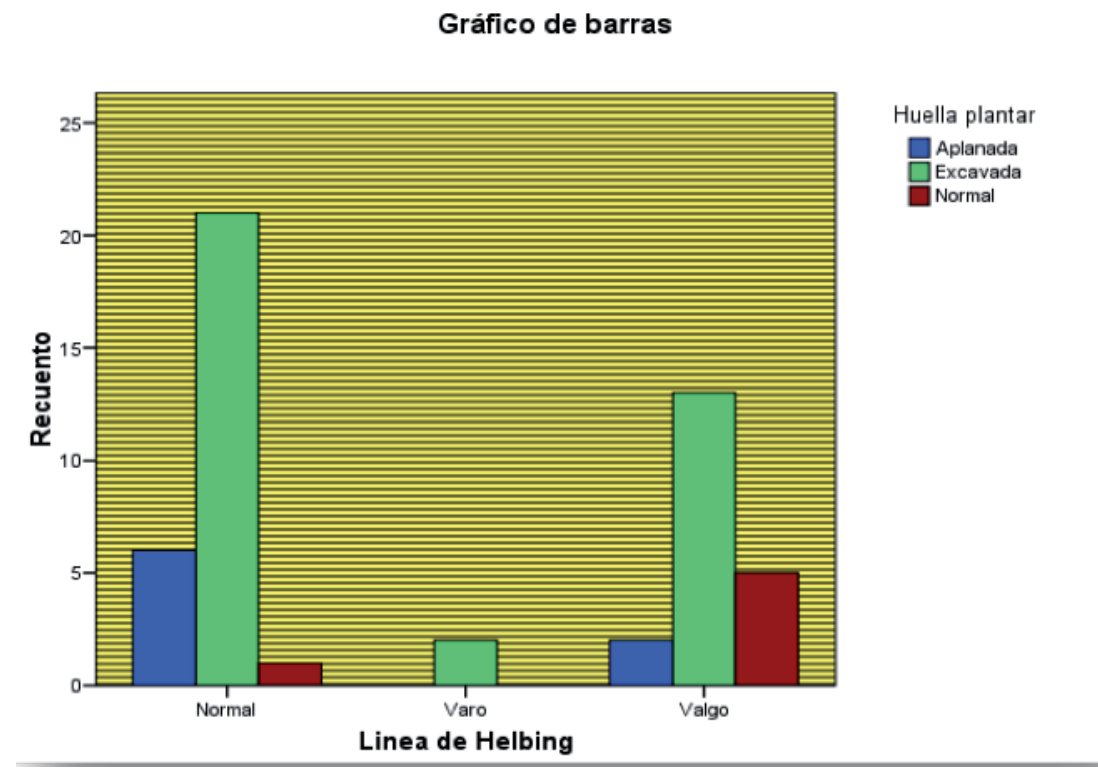

Figura 5: Gráfico comparativo de Línea de Helbing- huella plantar. 
En 1960, Villadot realizó un estudio examinando 273 pies en los que el resultado fue un $5,9 \%$ de tipo cuadrado, un $72,9 \%$ de tipo egipcio y un $21,2 \%$ de tipo griego ${ }^{50}$.

Otros estudios realizados por Sant y Metres y comparando con diferentes Estados como son: Canadá (Cousin), Francia (Lelievre, Dubois y Manet) y Méjico (Calderón) se obtuvieron las siguientes proporciones ${ }^{50}$ :
Al comparar nuestros resultados con los diferentes estudios realizados podemos comprobar que nuestra estadística está más próxima a las americanas, por lo que no coincidimos con la propuesta de Villadot que decía que: "En las estadísticas europeas, a diferencia de las americanas, se puede observar una coincidencia en la mayor proporción de pies egipcios, lo que nos lleva a cuestionarnos si

Tabla 5. Resultados de la frecuencia de laxitud ligamentosa en el estudio.

\begin{tabular}{cccc} 
& & Frecuencia & Porcentaje \\
& NO & 42 & 84,0 \\
Válidos & SI & 8 & 16,0 \\
\cline { 2 - 4 } & Total & 50 & 100,0 \\
\hline
\end{tabular}

Tabla 6. Comprobación de la frecuencia laxitud ligamentosa con respecto al sexo.

Tabla de contingencia sexo del individuo * Laxitud ligamentosa

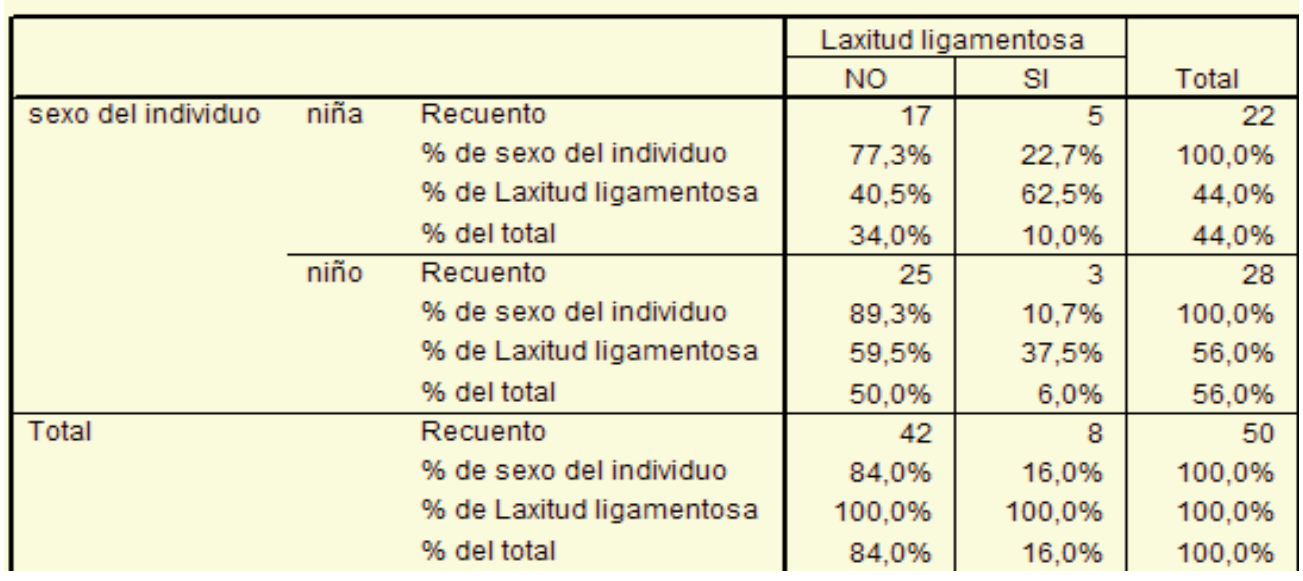

\begin{tabular}{|c|c|c|c|c|c|}
\hline \multicolumn{6}{|c|}{ Pruebas de chi-cuadrado } \\
\hline & Valor & gl & $\begin{array}{l}\text { Sig. asintótica } \\
\text { (bilateral) }\end{array}$ & $\begin{array}{l}\text { Sig. exacta } \\
\text { (bilateral) }\end{array}$ & $\begin{array}{l}\text { Sig. exacta } \\
\text { (unilateral) }\end{array}$ \\
\hline Chi-cuadrado de Pearson & $1,323^{b}$ & 1 &, 250 & \multirow{5}{*}{, 277} & \multirow{5}{*}{, 223} \\
\hline $\begin{array}{l}\text { Corrección por } \\
\text { continuidad }\end{array}$ &, 580 & 1 & .446 & & \\
\hline Razón de verosimilitudes & 1,317 & 1 &, 251 & & \\
\hline $\begin{array}{l}\text { Estadistico exacto de } \\
\text { Fisher }\end{array}$ & & & & & \\
\hline $\mathrm{N}$ de casos válidos & 50 & & & & \\
\hline
\end{tabular}




\section{Gráfico de barras}

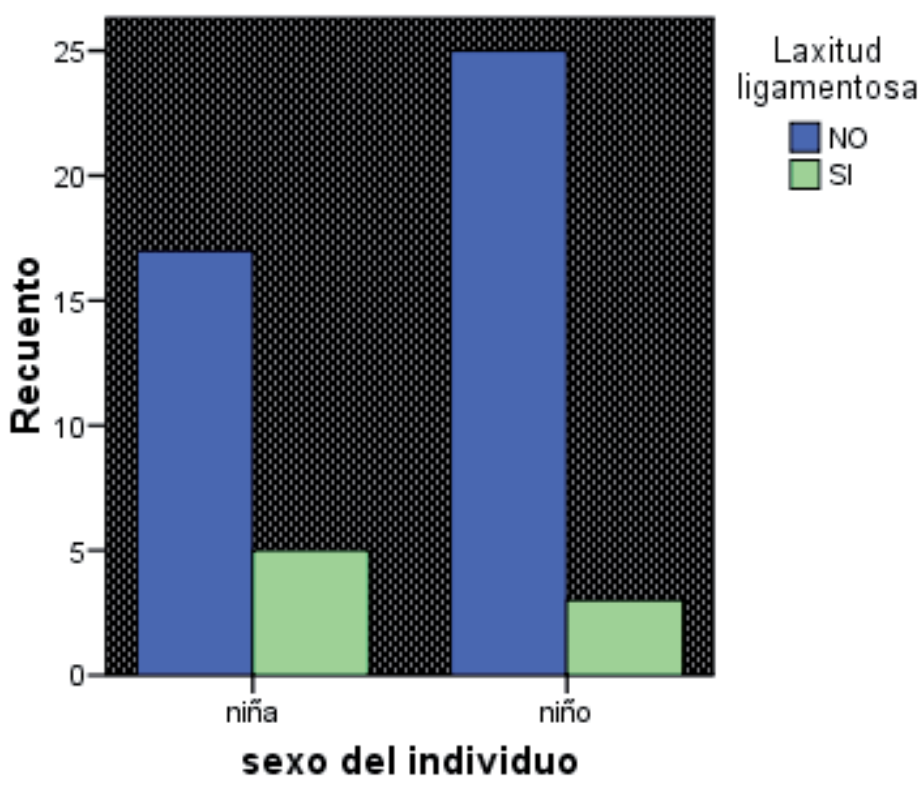

Figura 6. Gráfico laxitud ligamentosa-sexo.

Tabla 7. Resultados del pie egipcio.

\begin{tabular}{|cc|}
\hline Estadística canadiense & $37 \%$ \\
\hline Estadística de Villadot & $69 \%$ \\
\hline Estadística de Lelievre et al & $64,2 \%$ \\
\hline Estadística mejicana & $42,4 \%$ \\
\hline
\end{tabular}

Tabla 8. Resultados del pie griego.

\begin{tabular}{|cc|}
\hline Estadística canadiense & $40 \%$ \\
\hline Estadística de Villadot & $22,3 \%$ \\
\hline Estadística de Lelievre & $14,8 \%$ \\
\hline Estadística mejicana & $36,4 \%$ \\
\hline
\end{tabular}

Tabla 9. Resultados del pie cuadrado.

\begin{tabular}{|cc|}
\hline Estadística canadiense & $22 \%$ \\
\hline Estadística de Villadot & $\mathbf{8 , 7 \%}$ \\
\hline Estadística de Lelievre et al & $20 \%$ \\
\hline Estadística mejicana & $36,4 \%$ \\
\hline
\end{tabular}


existe una diferencia genotípica entre los dos continentes" $" 50$.

Excepto en el pie griego que obtenemos valores más aproximados a los estudios europeos.

En cuanto al tipo de pie nuestra estadística muestra que un $72 \%$ de la población estudiada muestra una huella excavada seguida de un $16 \%$ referida a la huella aplanada y un $12 \%$ correspondiente a una huella normal.

Combinada con la variable sexo podemos concluir que las huellas más frecuentes son las excavadas, siendo más predominante en el sector niñas con un $81,85 \%$ frente a un $64,28 \%$ en los niños.

Para José Ramos Galván, sin embargo, en el estudio llevado a cabo en Sevilla obtuvo que el $31,9 \%$ de la población tiene una huella plana frente a un $2,5 \%$ con una huella cava y, referido al sexo, los niños presentaban un 3,3\% frente a un $2,4 \%$ en las niñas, por lo que no estamos de acuerdo con este estudio ${ }^{16}$.

El pie plano es una condición fisiológica durante los 3 primeros años de vida ${ }^{43}$.Un estudio realizado en Asunción en el año 2008 muestra la prevalencia del pie plano en un $32,3 \%$ y va decreciendo conforme aumenta la edad del niño y está relacionado con otros autores como son Hernández Corvo o Espinoza-Navarro ${ }^{42,51}$.

R. Wózniacka, A. Bac et al en un reciente estudio aseguran que el pie plano se presenta en menos de 10 casos en la edad de 7 a 11 años, mientras que el pie cavo es el más frecuente indicando que presenta una mayor predominancia en las niñas ${ }^{52}$.

Nikolaidou, en el estudio realizado en el 2006 dijo que aproximadamente para el 85$88 \%$ el pie era normal ${ }^{53}$.

Al comparar nuestros resultados con los diferentes estudios realizados a lo largo de los años, estamos de acuerdo con R.Wózniacka porque el $72 \%$ de nuestra muestra presentaba una huella excavada y en las niñas existe un mayor porcentaje.

Según el estudio realizado por Raquel Sánchez Rodríguez y colaboradores, los pies pronados presentan una asociación con una mayor presión bajo la $1^{\circ}$ cabeza metatarsal frente a los pies supinados que tienen su mayor presión bajo la $5^{\circ}$ cabeza $^{2,26}$.

Hay que tener en cuenta que las personas con pies cavos son más propensas a lesionarse ${ }^{54}$.

Otros estudios realizados por Revenga y Espinoza, muestran una relación entre la aparición de pie plano con un valgo de retropié ${ }^{24,51}$.

Sin embargo, según nuestros resultados obtenidos para la huella excavada existe una mayor predominancia de retropié normal con 21 casos frente al retropié valgo con 16 personas.

En referencia al tipo de calzado muestran que el 56\% de la muestra utilizan zapatillas deportivas, seguido del $40 \%$ que utilizan botas.

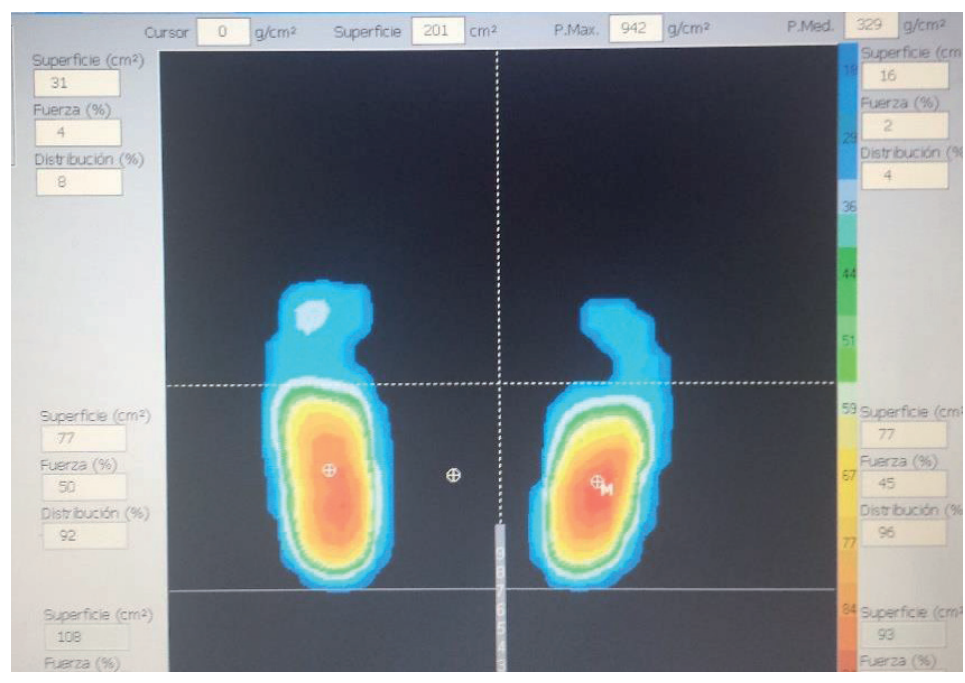

Figura 7. Huella aplanada de una niña de 9 años $(68,9 \mathrm{~kg})$. 
Al combinarlos con el sexo, hay una diferencia entre varones y hembras, ya que las deportivas son utilizadas por el $78,6 \%$ de niños y las botas usadas por el $63,6 \%$ habiendo una diferencia de calzado entre ambos.

Además, el $56 \%$ de la población de estudio no presenta una buena sujeción y, por lo tanto, no tienen un buen calzado fisiológico.

Diferentes estudios, como el realizado por José Ramos Galván, concluyen con que la mayoría de la población (89\%) utiliza un calzado deportivo y que tan solo el $8 \%$ utiliza un calzado fisiológico ${ }^{16}$.

Al estudiar en nuestra población la laxitud ligamentosa obtenemos que un $84 \%$ de la muestra no la presentan frente a un $16 \%$ que si la padecen.

En cuanto al sexo es más frecuente en niñas con un $22,8 \%$.

Gumà et al la sitúan entre un $15-25 \%$ ya que afecta en mayor medida al género femenino. Datos similares fueron obtenidos por Van der Giessen et al con un 20,8\%, Jonsson con un $27,7 \%$ y Gedalia y Press con un $12 \%{ }^{12,55}$.

Otros autores como De Cunto et al obtuvo el porcentaje de 37,3\% y Arroyo y colaboradores un $34 \%$, a lo cual estamos en desacuerdo ${ }^{12}$.

En los estudios referidos al sexo, todos los autores están de acuerdo en que las mujeres tienen una mayor prevalencia a padecer este síndrome ${ }^{12}$.

Relacionando la huella plantar con el peso corporal, en nuestro estudio solo había 2 personas que tenían un peso mayor al de la media $(37,3 \mathrm{~kg})$.

Por lo que estamos de acuerdo con el estudio realizado por Riddiford-Harland et al quienes hallaron mayores áreas plantares en sujetos obesos de 8 a 9 niños frente a sujetos no obe$\operatorname{sos}^{56}$.

También el estudio realizado por Mickle et al asocia presentar el pie plano a un mecanismo de sobrecarga continuada en el tiempo influyendo el peso $^{57}$.

Con este estudio hemos podido comprobar la influencia de diversos factores, tanto intrínsecos como extrínsecos sobre la cinética de la marcha ${ }^{1}$.

Nos planteamos en un futuro $q$ en nuestro estudio existe la necesidad de realizar un estudio estático y dinámico de la huella plantar ya que, durante la marcha, un pie patológico puede comportarse como un pie con huella normal'2.

Para mejorar el estudio de la presión plantar, se recomienda en estudios posteriores utilizar la técnica de 3D con un scanner de estructuras óseas del pie para identificar patologías óseas y plantares de los sujetos de forma más certera ${ }^{3}$.

Para llevar a cabo un estudio más completo, proponemos implementar una estructura mecánica para el análisis de la estabilidad y de la marcha con los últimos avances ${ }^{59,60}$.

Otra línea de estudio para el futuro sería el realizar un estudio teniendo en cuenta variables como la asimetría de los miembros inferiores en niños ${ }^{61}$ y realizar diferentes mediciones de las presiones plantares, distintos parámetros.

\section{CONCLUSIONES}

Nuestra primera línea de estudio consistió en averiguar qué tipo de huella plantar es más frecuente en niños de 8 a 10 años de edad. Tras realizar un minucioso estudio llegamos a la conclusión que la huella plantar más frecuente en niños de 8 a 10 años de edad en el área de Plasencia es la huella excavada con un $72 \%$, seguida de la huella aplanada con un $16 \%$ y en último lugar, la huella normal con un $12 \%$.

Después propusimos una serie de objetivos específicos a los que concluimos de la siguiente manera:

- Que al "Comprobar si existe influencia del peso en la huella plantar de cada niño" hemos llegado a la conclusión que aquellos sujetos que presentaban un mayor peso corporal, al realizarle el estudio en estática comprobamos que poseían una huella aplanada.

- Que al "Comprobar si utilizan un buen calzado con sujeción para las distintas actividades a realizar" podemos afirmar que el $56 \%$ de la muestra no tienen un buen calzado para llevar a cabo distintas actividades.

- Que valorando el tipo de retropié que presentan, obtenemos que el 56\% de los niños tienen un retropié normal, seguido de un retropié valgo perteneciente al $40 \%$ y tan sólo el 4\% de la muestra estudiada presentaba el retropié varo. 
- Que al "Comprobar qué tipo de formula digital es más frecuente" encontramos que es el pie egipcio con un $40 \%$ seguido de un pie cuadrado con un $34 \%$ y un pie griego con un $26 \%$.

- Que al "Comprobar si existe relación entre la huella plantar y línea de Helbing” llegamos a la conclusión que la mayoría de los niños estudiados que presentaban una huella excavada tenían un retropié normal, confirmándose así las frecuencias obtenidas en los estudios individualizados de las variables.
- Que estudiando la "hiperlaxitud articular" podemos decir qué la hiperlaxitud articular es una enfermedad que pese a algunos autores, en niños entre 8 y 10 años, es frecuente solamente en un $16 \%$ frente al $84 \%$ que no la padecen.

- Que con respecto al sexo y la hiperlaxitud articular, obtenemos que es más frecuente en niñas con un $22,7 \%$ que en niños con un $10,8 \%$.

- Que finalmente, los niños de la muestra con hiperlaxitud articular no referían ningún tipo de dolor musculoesqueletico.

\section{BIBLIOGRAFIA}

1. Collado Vázquez S. Análisis de la marcha humana con plataformas dinamométricas. Influencia del transporte de carga (tesis doctoral), Madrid, Universidad Complutense de Madrid, 2002.

2. Díaz CA, Torres A, Ramírez JI, García LF, Álvarez N. Descripción de un sistema para la medición de las presiones plantares por medio del procesamiento de imágenes Fase I, Revista EIA, 2006; 6:43-55.

3. Melgarejo Pinto VM, Moreno Rivera IT, Guzmán Sierra AJ, Hoyos Doria DP, Pacheco Tamayo E. Presión plantar: Estudio comparativo en estudiantes universitarios, Revista Actividad Física y Desarrollo Humano, 2013; 5(1):127-132.

4. Hernández Guerra RH. Prevalencia del pie plano en niños y niñas en las edades de 9 a 12 años. Revista Internacional de Medicina y Ciencias de la Actividad Física y el Deporte.2006; 6(23):165-172.

5. Ebri JR. El pie infantil: crecimiento y desarrollo. Deformidades más frecuentes: pie doloroso, Pediatr Integral 2002; 6(5):431-452.

6. Zurita MF, Cabello MD. Influencia del pie en la estática, marcha y otras habilidades en escolares de 6 a 12 años. Lect. Educ. Fís. Deportes, 2002; 8(51).

7. Collado Vázquez S. La marcha: Historia de los procedimientos de análisis, Revista de la Facultad de Ciencias de la Salud BIOCIENCIAS, 2004; 2:1-15.

8. Henry Osorio J, Hernando Valencia M. Bases para el entendimiento del proceso de la marcha humana, Arch Med ,2013; 13(1):88-96.

9. Martin Casas P. Análisis clínico, baropodométrico y neuromadurativo de los niños con marcha de puntillas idiopática en edad preescolar (tesis doctoral), Madrid, UNIVERSIDAD COMPLUTENSE DE MADRID. Escuela Universitaria de Enfermería, Fisioterapia y Podología, 2012.

10. Perucho Pont C, Pérez Mesquida ME, Guirao Cano LI, Pleguezuelos Cobo E. Síndrome de hiperlaxitud articular. A propósito de un caso. Rehabilitación. 2010; 44(2):180-182.

11. Martínez Larrarte JP, Suarez Martín R, Menéndez Alejo F, López Cabrejas G. El Síndrome de hiperlaxitud articular en la práctica clínica diaria, Órgano oficial de la Sociedad Cubana de Reumatología y el Grupo Nacional de Reumatología,2013; 15(1).

12. De Inocencio Arocena J, Ocaña Casas I, Benito Ortiz L. Laxitud articular: prevalencia y relación con dolor musculosquelético, An Pediatr. 2004; 61(2):162-166.

13. Laguna Nieto M, Alegre LM, Aznar Laín S, Abián Vicén J, Martín Casado L, Aguado Jódar X. ¿Afecta el sobrepeso a la huella plantar y al equilibrio de niños en edad escolar?, Apunts Med Esport. 2010;45(165):9-16.

14. Hurtado Padilla A. Uso de la baropodometría, Ortho-tips, 2006; 2(4):255-261.

15. Lara Dieguez S, Lara Sánchez AJ, Zagalaz Sánchez ML, Martínez-López EJ. Análisis de los diferentes métodos de evaluación de la huella plantar, Retos. Nuevas tendencias en Educación Física, Deporte y Recreación, 2011; 19: 49-53.

16. Ramos Galván J. Detección precoz y confirmación diagnóstica de alteraciones podológicas en población escolar (tesis doctoral), Universidad de Sevilla, Sevilla, 2007. 
17. Salleras Sanmartí L. Educación sanitaria: principios, métodos y aplicaciones, $3^{\circ}$ ed, Madrid, Díaz de Santos SA, 1990: 13-15.

18. Sierra Landines LM, Mosquera Ochoa D. EL CUERPO COMO FUNDAMENTO PARA EL DISEÑO DE VESTUARIO: Estudio morfológico y anatómico del cuerpo humano a través de la biomecánica y la ergonomía (tesis doctoral), Universidad Pontificia Bolivariana, Facultad de diseño de Vestuario, Medellín, 2013:19-23.

19. Root ML, Orien WP, Weed JH, Huges RJ. Exploración Biomecánica del pie. Madrid: Ortocen; 1991:1.

20. Kirby KA. Biomechanics of the Normal and Abnormal Foot. J Am Podiatr Med Assoc 2000; 90: 3034.

21. Leopoldo F, Yabar E, Daniel O, García R, Cristian Cóndor C, Emilio Villavicencio Ch. Estudio para el diseño de un Sistema Baropodométrico Electrónico, Universidad Tecnológica del Perú, 2008;10: 1-4.

22. Céspedes T, Dorca A. Aprendamos a conocer y a cuidar nuestros pies. Fundación para la Diabetes. Anuario de la Diabetes 2003.http://www.fundaciondiabetes.org/div/articulos/pie_diabetico/pie_diabetico03.htm [Fecha de consulta: 11/02/2014].

23. Goldcher A, Martí VN, Ferrer JMA. Podología. Paris: Masson; 1992

24. Revenga-Giertych C, Bulo-Concellón MP. El pie plano valgo: evolución de la huella plantar y factores relacionados, Rev Ortop Traumatol. 2005; 49(4):271-280.

25. Echarri JJ, Forriol F. Desarrollo de la morfología de la huella plantar en niños congoleños y su relación con el uso de calzado, Rev Ortop Traumatol 2003; 47(6):395-399.

26. Sánchez Rodríguez R, Martínez-Nova A, Escamilla-Martínez E, Pedrera-Zamorano JD. Can the Foot Posture Index or their individual criteria predict dynamic plantar pressures?, Gait \& Posture,2012;36: 591-595.

27. Collado-Vázquez S, Carrillo JM. Balzac y el análisis de la marcha humana, Neurología. 2012.

28. Collado Vázquez S. Plataformas dinamométricas. Aplicaciones, Revista de la Facultad de Ciencias de la Salud BIOCIENCIAS,2005;3

29. Morton D. The human foot. New York: Columbia University Press, 1948.

30. Villadot A. Biomecánica: estática y exploración. Patología del antepié.4º ed, Barcelona: Toray .1998; 29-54.

31. Padovani, Villadot Perice R, Álvarez Goenaga F Patología del pie calzado. Jano, 1995; 49 (129); 98 102.

32. Arcan M, Brull MA. A fundamental characteristic of the human body and foot, the footground pressure pattern. Journal of Biomechanics, 1976; 9: 453-457.

33. Gedalia A, Press J. Articular symptoms in hypermobile children: A prospective study. J Pediatr 1991; 119:944-6.

34. Mikkelsson M, Salminen JJ, Kautiainen H. Joint hypermobility is not a contributing factor to musculoskeletal pain in pre-adolescents. J Rheumatol 1996; 23:1963-7.

35. De Cunto CL, Moroldo MB, Liberatore DI, Imach E. Hiperlaxitud articular: estimación de su prevalencia en niños en edad escolar. Arch Argent Pediatr 2001; 99:105-10.

36. Collazo Vázquez S, Pascual Gómez F, Ảlvarez Vadillo A, Rodríguez Rodríguez LP. Análisis de la marcha. Factores moduladores, Revista de la Facultad de Ciencias de la Salud, BIOCIENCIAS, 2003; $1: 1-22$.

37. López Olmedo J. Exploración ortopédica infantil básica, Pediatr Integral 2010; 14(7): 541-547.

38. López Olmedo J. Exploración ortopédica básica, Pediatr Integral 2006; 10(7):475-481.

39. Moreno JL. Podología general y Biomecánica. Barcelona: Masson; 2003.

40. Villadot A. Quince lecciones sobre patología del pie, Springer-Verlag Ibérica, 2000; 13.

41. Sastre Fernández S. Fisioterapia del pie. Podología física, Edicions Universitat Barcelona,1991;1:39.

42. Zárate Barchello A, Pereira López MA, Ibarrola Zárate J, Kikuchi A, Sanabria L. Prevalencia de pie plano en niños escolares de Asunción y Gran Asunción, en el año 2008, An. Fac. Cienc. Méd ,2009; 42(2):13-18.

43. Parra García J, Bueno Sánchez A. El pie plano; las recomendaciones del traumatólogo infantil al pediatra, Rev Pediatr Aten Primaria.2011; 13:113-125.

44. Segura EJ. Pie cavo. Rev Esp Podol 2001; 12(4): 234-236.

45. Colección práctico profesional. Profesiones sanitarias: Tratamiento fisioterápico en Pediatría, editorial Eduforma, 2006; 71.

46. Maure Ternia F. Tratado de podología estudio del desequilibrio en el esqueleto: causas y consecuencias, $1^{\circ} \mathrm{ed}$, editorial Jims SA, 1997:36-37. 
47. Instituto Biomecánico de Valencia. El pie calzado: Guía para el asesoramiento en la selección del calzado infantil,1999.

48. Lelièvre J, Lelièvre JF. Patología del pie.4º ed,Barcelona:Masson; 1982.

49. Hidalgo Ruiz S, Alonso Tajes F, Rosende Bautista C,García Blázquez. FM, Martínez Nova A, Sánchez Rodríguez R. Estudio de la altura recomendada del tacón, El Peu 2005; 25(2):73-78.

50. Villadot Perice A. Patología del antepie, $4^{\circ}$ ed, Barcelona ,2001; 2-3.

51. Espinoza-Navarro O, Olivares UM, Palacios NP, Robles FN. Prevalencia de anomalías de pie en niños de enseñanza básica de entre 6 a 12 años, de colegios de la ciudad de Arica-Chile. Int. J. Morphol.2013; 31(1):162-168.

52. Woźniacka R, Bac A, Matusik S, Szczygieł E, Ciszek E. Body weight and the medial longitudinal foot arch:high-arched foot, a hidden problem?, Eur J Pediatr,2013;172:683-691.

53. Nikolaidou ME, Boudolos KD. A footprint-based approach for the rational classification of foot types in young schoolchildren, The Foot, 2006; 16: 82-90.

54. Zurita Ortega F, Martínez Martínez A, Zurita Ortega A. Influencia de la tipología del pie en la actividad físico deportiva, fisioterapia, 2007; 29(2):74-79.

55. Gumà M, Olivé A, Holgado S, Casado E, Roca J, Forcada J, et al. Una estimación de la laxitud articular en la consulta externa. Rev Esp Reumatol. 2001; 28:298-300.

56. Riddiford-Harland DL, Steele JR, Storlien LH. Does obesity influence foot structure in prepubescent children? Int J Obes.2000; 24:541-544.

57. Mickle KJ, Steele JR, Munro BJ. The feet of overweight and obese young children: Are they flat or fat? Obesity. 2006; 14:11.

58. Elvira JLL, Vera-García F.J, Meana M, García JA. análisis biomecánico del apoyo plantar en la marcha atlética. relación entre la huella plantar, ángulos de la articulación subastragalina y presiones plantares, Motricidad. European Journal of Human Movement, 2008: 20, 41-60.

59. Olivera Oliva R. Diseño de una plataforma dinamométrica para el cálculo del centro de presiones utilizando galgas extensiométricas (tesis doctoral),Pontificia Universidad Católica del Perú, Lima, 2012.

60. Berdejo-del-Fresno D, Lara Sánchez AJ, Martínez-López EJ, Cachón Zagalaz J,Lara Diéguez S. Alteraciones de la huella plantar en función de la actividad física realizada, Rev.int.med.cienc.act.fís. deporte, 2013; 13(49):19-39.

61. Diop M, Rahmani A,Belli A, Gautheron V,Geyssant A, Cottalorda J. Influence of speed variation and age on the asymmetry of ground reaction forces and stride parameters of normal gait in children, Journal of Pediatric Orthopaedics B, 2004;13:308-331.

62. Mickle KJ, Steele JR, Munro BJ. The feet of overweight and obese young children: are they flat or fat? Obesity (Silver Spring). 2006; 14: 1949-1953.

63. Sperotto F, Balzarin M, Parolin M, Monteforte N, Vittadello F, Zulian F.Joint hypermobility, growing pain and obesity are mutually exclusive as causes of musculoskeletal pain in schoolchildren.Clin Exp Rheumatol. 2014 Jan-Feb; 32 (1): 131-6.

64. Jiménez-Ormeño E, Aguado X, Delgado-Abellán L, Mecerreyes L, Alegre LM.Foot morphology in normal-weight, overweight, and obese schoolchildren. Eur J Pediatr. 2013; 172: 645-652.

65. Vergara AE, Serrano SRF, Correa PJR, Molano AC, Guevara OA. Prevalence of flatfoot in school between 3 and 10 years. Study of two different populations geographically and socially. Colomb Med. 2012; 43: 141-146.

66. Sperotto F, Brachi S, Vittadello F, Zulian F. Musculoskeletal pain in schoolchildren across puberty: a 3-year follow-up study. Pediatr Rheumatol Online J. 2015 May 15; 13:16. 